\title{
MEASURABILITY OF FUNCTIONS IN PRODUCT SPACES
}

\author{
KOHUR GOWRISANKARAN
}

\begin{abstract}
Let $f$ be a function on a product space $X \times Y$ with values in a separable metrizable space such that it is measurable in one variable and continuous in the other. The joint measurability of such a function is proved under certain conditions on $X$ and $Y$.
\end{abstract}

Let $X$ and $Y$ be Hausdorff topological spaces. Let $f$ be a complex valued function on the product space $X \times Y$ such that $f(\cdot, y)$ is Borel measurable on $X$ for every $y \in Y$ and $f(x, \cdot)$ continuous on $Y$ for every $x$ in $X$. The problem of proving measurability of $f$ on $X \times Y$, as a function of the variables together is of interest and has occupied the attention of many mathematicians ([2], [3], [3 $\left.\left.{ }^{\mathrm{bis}}\right],[5],[6]\right)$. For instance, in [2, p. 122], it is proved that if $Y$ is a locally compact separable metric space (and $X$ any measure space, not necessarily a topological space) then $f$ is jointly measurable. (See also [3].) In a recent paper, while considering the iterated fine limits of holomorphic functions [1], we had to prove the measurability of functions of the form $f$ as above, but with values in a separable metric space where neither $X$ nor $Y$ is necessarily locally compact. Our proof in this case carries over to more general situations. We state below the main results.

THEOREM 1. Let $X$ and $Y$ be Hausdorff topological spaces such that every compact subspace of $Y$ is metrizable. Let $\mu$ and $\nu$ be any two Radon measures on $X$ and $Y$ respectively [4]. Let $f: X \times Y \rightarrow A$, where $A$ is a separable metrizable space, be such that it is $\mu$-measurable on $X$ for every $y \in Y$ and continuous on $Y$ for every $x \in X$. Then, $f$ is $\mu \times v$-Lusin measurable.

THeOREM 2. Let $(X, \tau)$ be a measurable space (i.e. a set $X$ with a $\sigma$ algebra $\tau$ of subsets of $X)$; and $Y$ a Suslin space. Let $B$ be the $\sigma$-algebra of all measurable subsets for a locally finite measure $\mu$ on the Borel $\sigma$ algebra of $Y$. Then, a function $f$ on $X \times Y$ with values in a separable metrizable space $A, \tau$-measurable for every $y \in Y$ and continuous on $Y$ for each $x \in X$, is $\tau \times B$-measurable on $X \times Y$.

Received by the editors September 15, 1970 and, in revised form, April 6, 1971. AMS 1969 subject classifications. Primary 2820.

Key words and phrases. Measurability, product spaces, Radon measures, Suslin space, Polish space.

(c) American Mathematical Society 1972 
We are thankful to the referee for pointing out some of the references to earlier work on the topic.

Before proving the results, we recall

Definition 1. A Radon measure $\mu$ on a Hausdorff topological space $X$ is a positive measure defined on the $\sigma$-algebra of Borel subsets of $X$ (generated by the open sets) such that (i) $\mu$ is locally finite and (ii) for every Borel subset $B$ of $X, \mu(B)=\sup \{\mu(K)$ : compact $K \subset B\}$.

Definition 2. Let $X$ and $Y$ be Hausdorff topological spaces and $\mu$ a Radon measure on $X$. A function $f: X \rightarrow Y$ is said to be $\mu$-Lusin measurable (resp. $\mu$-Borel measurable) if, for every $\varepsilon>0$ and any compact set $K \subset X, \exists$ a compact set $C \subset K$ satisfying (i) $\mu(C)>\mu(K)-\varepsilon$ and (ii) $f / C$ is continuous (resp. $f^{-1}(B)$ is $\mu$-measurable for every Borel subset $B$ of $Y$ ).

\section{Proof of Theorem 1.}

Case I. Let $Y$ be compact metric and $f$ a real valued function. We deduce from [2, p. 122] that $f$ is $\mu \times \nu$-Borel measurable. However such a function is necessarily $\mu \times \nu$-Lusin measurable [4].

Case II. Let $Y$ be as in the theorem, but $f$ a real valued function. Let $\mu$ and $\nu$ be as in the statement of the theorem and $C \subset X \times Y$ a compact set. Let $K=p_{1}(C) \times p_{2}(C)$ where $p_{1}$ and $p_{2}$ are respectively the projections onto $X$ and $Y$. Now, $p_{2}(C)$ is compact and metrizable (hypothesis) and hence, by the Case $I, f$ restricted to $K$ is $\mu \times \nu$-Lusin measurable. This completes the proof in this case.

Case III. General case. Let $d$ be a metric on $A$, compatible with its topology. For $z_{0} \in A, \varepsilon>0$, let $B\left(z_{0}, \varepsilon\right)$ be the open ball of radius $\varepsilon$ and centre at $z_{0}$. Now, $(x, y) \rightarrow d\left[z_{0}, f(x, y)\right]$ is easily seen to be a function of the nature considered in Case II and hence is $\mu \times \nu$-Lusin measurable on $X \times Y$. This implies the $\mu \times \nu$-Borel measurability of $f$. Hence, $\left\{(x, y): d\left(z_{0}, f(x, y)\right)<\varepsilon\right\}$ is $\mu \times \nu$-measurable. However, this set is the same as $f^{-1}\left(B\left(z_{0}, \varepsilon\right)\right)$. This is true for every $\varepsilon>0$ and $z_{0} \in A$. Hence, for a base of open sets $V \subset A$, viz., all open balls, $f^{-1}(V)$ is $\mu \times \nu$-measurable. But every open set of $A$ is a countable union of open balls; we deduce that $f^{-1}(V)$ is $\mu \times \nu$-measurable for every open set $V \subset A$. Now, by standard argument we get that $f$ is $\mu \times \nu$-Borel measurable. However, since $A$ is separable and metrizable, this implies the $\mu \times v$-Lusin measurability of $f$ [4, Chapter I]. This completes the proof of the theorem.

Now we recall

Definition 3. A Suslin space is a Hausdorff topological space which is the continuous image of a Polish (separable, complete metrizable) space.

Proof of Theorem 2. Let us suppose first of all that $Y$ is a Polish space and $f$ real valued and $B$ the Borel $\sigma$-algebra of $Y$. For every $k \in N$, let the open balls $B_{n, k}, n \in N$ of radius $1 / k$ and centres at $y_{n, k}$ cover $Y$. Let $a \in R$. 
Then

$$
\{(x, y): f(x, y)>a\}=\bigcup_{m \geqq 1} \bigcup_{l \geqq 1} \bigcap_{k \geqq l} \bigcup_{n \geqq 1}\left[\left\{x: f\left(x, y_{n, k}\right)>a+\frac{1}{m}\right\} \times B_{n, k}\right] .
$$

For if $\left(x_{0}, y_{0}\right) \in$ L.H.S., then by the continuity of $f\left(x_{0}, \cdot\right)$ there exists an open ball of radius $2 / l_{0}$ and centre $y_{0}$ such that for every $y \in B\left(y_{0}, 2 / l_{0}\right)$, $f\left(x_{0}, y\right)>a+1 / m_{0}$ for some $m_{0}$. For every $k \geqq l_{0}$, if $y_{0} \in B_{n_{k}, k}$ with centre at $y_{n_{k}, k}$ then $\left(x_{0}, y_{0}\right) \in\left\{x: f\left(x, y_{n_{k}, k}\right)>a+1 / m_{0}\right\} \times B_{n_{k}, k}$ and this shows that L.H.S. $\subset$ R.H.S. On the other hand, if $\left(x_{0}, y_{0}\right) \in$ R.H.S., then $\exists$ some $m_{0}$ and $l_{0}$ such that for every $k \geqq l_{0}$, there is an integer $n_{k}$ such that $f\left(x_{0}, y_{n_{k}, k}\right)>a+1 / m_{0}$ and $y_{0} \in B_{n_{k}, k}$. Let $y_{k}^{\prime}=y_{n_{k}, k}$. Then, since the distance between $y_{k}^{\prime}$ and $y_{0}$ is less than $1 / k$ for every $k$, we get that $y_{k}^{\prime} \rightarrow y_{0}$. By the continuity of $f\left(x_{0}, \cdot\right)$ we deduce that $f\left(x_{0}, y_{0}\right) \geqq a+1 / m_{0}>a$. Hence the two sides are identical and we conclude that $\{(x, y): f(x, y)>a\}$ belongs to $\boldsymbol{\tau} \times \boldsymbol{B}$.

Now, in case $f$ is with values in a separable metrizable space, the $\boldsymbol{\tau} \times \boldsymbol{B}$ measurability of $f$ is proved exactly in the same way as in the general case (III) of Theorem 1.

To complete the proof, we have to consider the case when $Y$ is a Suslin space. Then $\exists$ a Polish space $P$ and a continuous onto mapping $\varphi$ of $P \rightarrow Y$. By what we have proved already the function $g(x, p)=f[x, \varphi(p)]$ on $X \times P$ is $\tau \times B^{\prime}$-measurable, where $\boldsymbol{B}^{\prime}$ is the Borel $\sigma$-algebra of $P$. However, there exists a mapping $\pi: Y \rightarrow P$ such that $\varphi \circ \pi$ is identity on $Y$ and $\pi^{-1}(B)$ is $\nu$-measurable for every locally finite measure $\nu$ on $Y$ for every $B \in B$ [4, Chapter II].

Now, let $\boldsymbol{\nu}$ be a locally finite Borel measure on $Y$ and $B$ the class of all $\nu$-measurable sets. Since, $I \times \pi:(X \times Y, \tau \times B) \rightarrow(X \times P, \tau \times B)$ and $I \times \varphi$ : $(X \times P, \tau \times \boldsymbol{B}) \mapsto(X \times Y, \tau \times \boldsymbol{B})$ are both measurable, where $I$ is the identity map on $X$, we deduce that $f=f \circ(I \times \varphi) \circ(I \times \pi)=g \circ(I \times \pi)$ is $\tau \times B$-measurable. The proof is complete.

REMARK. In case $Y$ is just a separable metrizable space (not necessarily complete) a function $f$ as in the hypothesis of the theorem is measurable where $\boldsymbol{B}$ is the Borel $\sigma$-algebra of $Y$.

THEOREM 3. Let $X$ be a set with a $\sigma$-algebra $\tau$ of subsets. Let $f: X \times$ $\boldsymbol{R} \mapsto A$ be $\tau$-measurable for every $y \in \boldsymbol{R}$ and right continuous on $\boldsymbol{R}$ for every $x \in X$, where $A$ is a separable metrizable space. Then $f$ is measurable with respect to $\tau \times \boldsymbol{B}$ where $\boldsymbol{B}$ is the Borel $\sigma$-algebra of $\boldsymbol{R}$.

Proof. As in the earlier theorems, it is enough to prove the theorem assuming that the values are taken on $\boldsymbol{R}$. Let, for every $k \in N, I_{n, k}$ be the interval $((n-1) / k, n / k]$ for $n \in Z$. Then as in the proof of Theorem 2 , it could be verified that 


$$
\begin{aligned}
\{(x, y): f(x, y)> & a\} \\
& =\bigcup_{m \geqq 1} \bigcup_{l \geqq 1} \bigcap_{k \geqq l} \bigcup_{n \in \mathbf{Z}}\left\{x: f\left(x, \frac{n}{k}\right)>a+\frac{1}{m_{0}}\right\} \times\left(\frac{n-1}{k}, \frac{n}{k}\right] .
\end{aligned}
$$

This shows that $f$ is $\tau \times \boldsymbol{B}$-measurable, completing the proof.

REMARK 1. A similar result is true if we have left continuity of $f(x, \cdot)$, for every $x \in X$, instead of right continuity.

REMARK 2. Some sort of continuity in at least one of the variables seems to play an important role in proving the above measurability properties. There is an example (due to Sierpiński [5]) of a function which is upper semicontinuous in each variable but not jointly measurable.

\section{BIBLIOGRAPHY}

1. K. Gowrisankaran, Iterated fine limits and iterated nontangential limits (to appear).

2. G. W. Mackey, Induced representations of locally compact groups. I, Ann. of Math. (2) 55 (1952), 101-139. MR 13, 434.

3. M. E. Mahowald, On the measurability of functions in two variables, Proc. Amer. Math. Soc. 13 (1962), 410-411. MR 25 \#1261.

$3^{\text {bis }}$. W. Rudin, Real and complex analysis, McGraw-Hill, New York, 1966, p. 150. MR 35 \#1420.

4. L. Schwartz, Rado measures on general topological spaces, Tata Institute of Fundamental Research Monographs, 1971 (to appear).

5. W. Sierpiński, Sur un probleme concernant les ensembles measurables superficiellment, Fund. Math. 1 (1920), 112-115.

6. H. D. Ursell, Some methods of proving measurability, Fund. Math. 32 (1939), 311-330.

Department of Mathematics, McGill University, Montreal, Quebec, Canada 